\title{
ARTICLE Supplementation of p40, a Lactobacillus rhamnosus GG- derived protein, in early life promotes epidermal growth factor receptor-dependent intestinal development and long-term health outcomes
}

\author{
Xi Shen ${ }^{1,2}$, Liping Liu' ${ }^{1}$, Richard M. Peek ${ }^{3}$, Sari A. Acra ${ }^{1}$, Daniel J. Moore ${ }^{4}$, Keith T. Wilson ${ }^{3,5}$, Fang He ${ }^{2}$, D. Brent Polk ${ }^{6,7}$ and Fang Yan ${ }^{1,3}$
}

The beneficial effects of the gut microbiota on growth in early life are well known. However, knowledge about the mechanisms underlying regulating intestinal development by the microbiota is limited. p40, a Lactobacillus rhamnosus GG-derived protein, transactivates epidermal growth factor receptor (EGFR) in intestinal epithelial cells for protecting the intestinal epithelium against injury and inflammation. Here, we developed p40-containing pectin/zein hydrogels for targeted delivery of p40 to the small intestine and the colon. Treatment with p40-containing hydrogels from postnatal day 2 to 21 significantly enhanced bodyweight gain prior to weaning and functional maturation of the intestine, including intestinal epithelial cell proliferation, differentiation, and tight junction formation, and IgA production in early life in wild-type mice. These p40-induced effects were abolished in mice with specific deletion of EGFR in intestinal epithelial cells, suggesting that transactivation of EGFR in intestinal epithelial cells may mediate p40-regulated intestinal development. Furthermore, neonatal p40 treatment reduced the susceptibility to intestinal injury and colitis and promoted protective immune responses, including IgA production and differentiation of regulatory $\mathrm{T}$ cells, in adult mice. These findings reveal novel roles of neonatal supplementation of probiotic-derived factors in promoting EGFR-mediated maturation of intestinal functions and innate immunity, which likely promote long-term beneficial outcomes.

Mucosal Immunology (2018) 11:1316-1328; https://doi.org/10.1038/s41385-018-0034-3

\section{INTRODUCTION}

The symbiotic relationship between the gut microbiota and the host contributes to maturation of the intestinal functions ${ }^{1}$ and immunity, ${ }^{2}$ resulting in enhanced growth and health in early life. Studies have shown that impaired development of the gut microbiota is related to undernutrition in children, ${ }^{3}$ while several diseases in children are associated with alteration of the gut microbiome, such as inflammatory bowel disease (IBD), including ulcerative colitis and Crohn's disease. ${ }^{4,5}$ Furthermore, exposure to microbes during early life has shown to have protective effects on IBD in adults, ${ }^{6}$ which indicates that the influence of the gut micobiota on childhood can persist into later stages of life. Thus, interventions aimed at manipulating the gut microbiota in early life may have the potential to promote growth during development and exert long-term health outcomes.

Probiotics are defined as "live microorganisms that, when administered in adequate amounts, confer a health benefit on the host". ${ }^{7}$ Clinical studies have revealed the beneficial effects of probiotics on the outcomes of infants and young children with infectious diarrhea ${ }^{8,9}$ and on prevention of antibiotic-associated diarrhea in children. ${ }^{9}$ Increasing evidence from animal studies supports administering probiotics at the early stage for promoting growth and long-term health outcomes. Mono-colonization of infant germ-free mice with Lactobacillus plantarum promotes juvenile growth during chronic undernutrition. ${ }^{10}$ Furthermore, neonatal colonization of conventionally raised mice with Lactobacillus rhamnosus GG (LGG) promotes growth, intestinal functional maturation, persistent IgA production, and decreased susceptibility to intestinal injury and colitis in adult mice, ${ }^{11}$ suggesting that LGG colonization has an impact on intestinal development and long-term health outcomes in adulthood.

Multiple mechanisms of probiotic action have been suggested; however, little is known about precise mode of probiotic action. ${ }^{12,13}$ Several factors derived from the gut microbiota, including probiotic bacteria, have been identified as functional effectors. p40 was originally isolated from LGG culture supernatant by our group. ${ }^{14}$ Production of this secretory protein is strainspecific. ${ }^{14}$ Functional analysis of $\mathrm{p} 40$ revealed that $\mathrm{p} 40$ preserves barrier function, suppresses cytokine-induced apoptosis, promotes mucin production in intestinal epithelial cells, and enhances $\lg \mathrm{A}$

\footnotetext{
${ }^{1}$ Department of Pediatrics, Division of Gastroenterology, Hepatology and Nutrition, Vanderbilt University Medical Center, Nashville, TN 37232, USA; ${ }^{2}$ West China School of Public

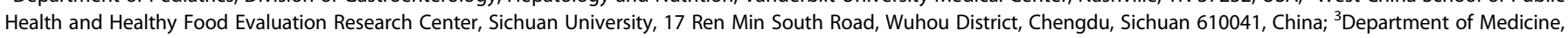

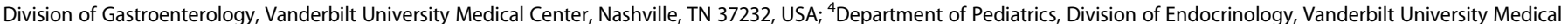

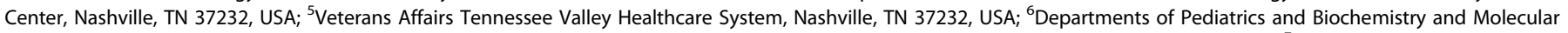

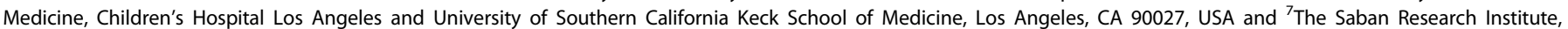
Children's Hospital Los Angeles, Los Angeles, CA 90027, USA
}

Correspondence: Fang Yan (fang.yan@vanderbilt.edu)

Received: 3 July 2017 Revised: 1 April 2018 Accepted: 15 April 2018

Published online: 6 June 2018 
production through up-regulation of the expression of a proliferation-inducing ligand (APRIL) in intestinal epithelial cells, ${ }^{14-17}$ thereby protecting the intestine from injury and inflammation in mice. ${ }^{17}$ Further studies demonstrated that transactivation of the epidermal growth factor receptor (EGFR) in intestinal epithelial cells through activation of a disintegrin and metalloproteinase domain-containing protein-17 (ADAM-17) for $\mathrm{HB}-\mathrm{EGF}$ release serves as a signaling mechanism for regulating these protective cellular responses by $\mathrm{p} 40 .^{18}$

As a member of the ErbB family of type 1 receptor tyrosine kinases, activation of EGFR stimulates a variety of downstream signaling pathways, such as Akt and mitogen-activated protein kinase, leading to several cellular responses, including cell proliferation, differentiation, migration, and survival. ${ }^{19,20}$ EGFR signaling is required for postnatal growth because insufficient EGFR signaling causes death in the perinatal period, and surviving mice die from hemorrhagic enteritis in the postnatal period. ${ }^{21}$ Studies have also shown that ErbB ligands, EGF ${ }^{22,23}$ and HB-EGF, ${ }^{24}$ are present in amniotic fluid and breast milk and are involved in stimulating intestinal epithelial cell growth. Growth and epithelial cell proliferation are enhanced by EGF-expressing Lactococcus lactis in mice at weaning. ${ }^{25}$ While some EGFR mutations are considered to be tumor promoters, EGF has shown therapeutic potential in human ulcerative colitis ${ }^{26}$ and EGFR activation ameliorates chronic inflammation, thus limiting colitis-associated tumorigenesis. ${ }^{27}$

Here we show that neonatal p40 supplementation promotes functional maturation of the intestine and stimulates persistent IgA production from early life to adulthood, in an EGFR-dependent manner. Adult mice with p40 supplementation in early life exhibit decreased susceptibility to intestinal injury and inflammation. Thus, these results broaden our understanding of the mechanisms underlying the effects of probiotics on intestinal development and immune responses in early life and long-term health outcomes.

\section{RESULTS}

Development of a hydrogel system for delivering p40 to the small intestine and the colon in neonatal mice

p40 used in this study was purified from LGG culture supernatant, as previously reported. ${ }^{14}$ The level of endotoxin in p40 isolates was $<0.03 \mathrm{EU} / \mu \mathrm{g}$ p40 protein. For protecting p40 from the harsh conditions in the gastrointestinal tract, such as the presence of proteases and an acidic environment in the stomach, a pectin and zein delivery system was generated in our previous study for specifically delivering p40 to the colon. ${ }^{17}$ This colon-specific delivery system requires the high concentration of pectin, such as $6 \% \mathrm{w} / \mathrm{v},{ }^{17}$ with the release of $\mathrm{p} 40$ depending on complete degradation of pectin by colonic microflora-produced pectinolytic enzymes in the colon. In this study, we decreased the concentration of pectin to $2 \% \mathrm{w} / \mathrm{v}$ to allow p40 release starting in the small intestine before pectin degradation in the colon. Hydrogels were coated with zein, which protects drugs from gastric acid by its hydrophobic nature. Zein is released by enzymes in the small intestine. We prepared pectin/zein hydrogels containing 0.5 and $1.0 \mu \mathrm{g}$ of p40 per hydrogel. Albumin-containing hydrogels and pectin/zein only hydrogels (without p40 and albumin) were used as negative controls. Hydrogels were prepared as thin sheets with the average size of $11 \mathrm{~mm}^{2}$ and could be melted into a semi-solid state after oral administration.

To assess p40 release in the gastrointestinal tract, 14-day-old mice were administered orally hydrogels containing NHSrhodamine-labeled p40 and euthanized at 2, 4, and $6 \mathrm{~h}$ after treatment. Mice receiving hydrogels without $p 40$ for $6 \mathrm{~h}$ were used as control. Tissues from the stomach, small intestine, and colon were examined. NHS-rhodamine-p40 was recovered from the small intestinal mucosal lysates with the amount decreasing from 2 to $6 \mathrm{~h}$ after gavage (Fig. 1a). In the colonic mucosal lysates, p40 was recovered at $6 \mathrm{~h}$ after gavage, with no significant amount of NHS-rhodamine-p40 identified at 2 and $4 \mathrm{~h}$ after gavage (Fig. 1a). Paraffin-embedded tissue sections were prepared for evaluating the delivery of NHS-rhodamine-p40 under a fluorescent microscope. NHS-rhodamine-p40 was found on the surface of the small intestine and the colon (Fig. 1b). Limited amounts of p40 were found in the stomach (data not shown).

We next examined whether delivery of p40 by this system stimulated transactivation of EGFR in intestinal epithelial cells in mice. Small intestinal and colonic epithelial cells were isolated from mice by sorting using an E-cadherin antibody. Phosphorylated proteins were immunoprecipated from cellular proteins and were used for western blot analysis of total EGFR. p40 treatment a
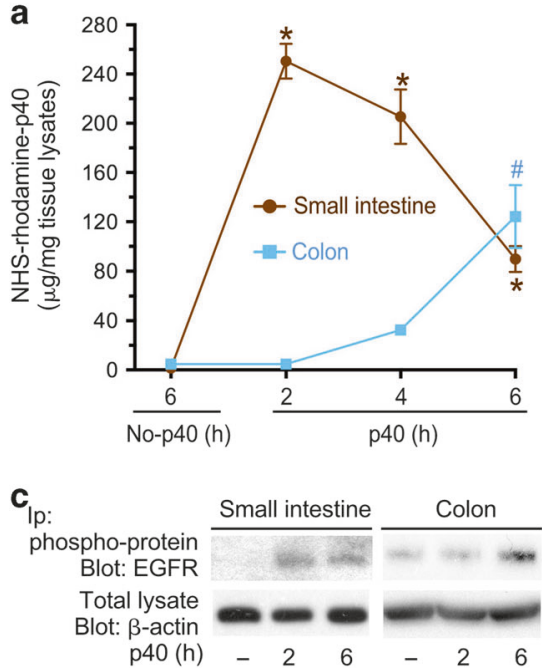

b Small intestine
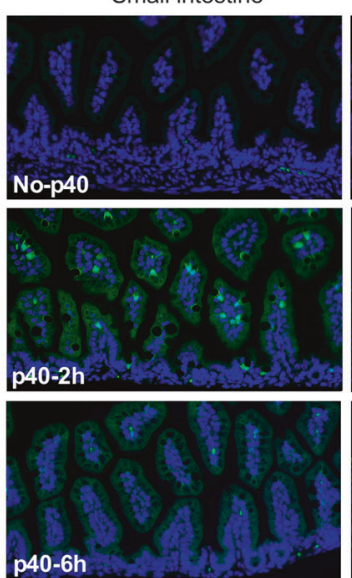

p40: green
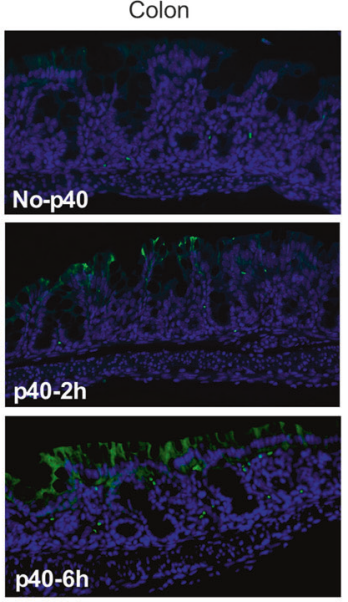

Fig. 1 Delivery of p40 to the small intestine and the colon using pectin/zein hydrogels. Fourteen-day-old mice were gavaged with hydrogels containing NHS-rhodamine-p40 at $10 \mu \mathrm{g} /$ mouse and hydrogels without p40 (no-p40) as control. Mice were euthanized at indicated times after gavage. Soluble proteins from small intestinal and colonic mucosal tissues were prepared for examining fluorescent intensity (a). The NHSrhodamine-p40 concentration in mucosal lysates was determined by comparing to the NHS-rhodamine-labeled concentration curve and presented as: $\mu \mathrm{g} \mathrm{p40/mg} \mathrm{tissue} \mathrm{lysates.} \mathrm{Paraffin-embedded} \mathrm{tissue} \mathrm{sections} \mathrm{were} \mathrm{prepared} \mathrm{for} \mathrm{observing} \mathrm{NHS-rhodamine-p40} \mathrm{using} \mathrm{a} \mathrm{fluorescent}$ microscope (b). Tyrosine phosphorylated proteins in tissue lysates were immunoprecipited for western blot analysis of total EGFR. Total tissue lysates were used for western blot analysis with anti- $\beta$-actin antibody to confirm the equal amount of proteins used for immunoprecipitation. In $a_{1}^{*}$ and \# $p<0.05$ compared to the small intestine $\left(^{*}\right)$ and the colon $\left(^{*}\right)$ of the no-p40 group. $n=3-5$ mice for each group 
increased phosphorylation of EGFR, which represents EGFR activation status, in small intestinal and colonic epithelial cells (Fig. 1c).

These results suggest that this hydrogel system is able to deliver p40 to the small intestine and the colon and preserve the activity of p40 for transactivation of EGFR. Thus, this system was applied for administration of $\mathrm{p} 40$ to mouse pups in the reminder of this study.

p40 treatment accelerates growth and promotes proliferation, differentiation, and tight junction formation in intestinal epithelial cells during development in wild-type mice

Supplementation of probiotics to infants is becoming increasingly common. LGG supplementation in formula enhances extensively hydrolyzed casein formula-induced acquisition of tolerance in children with cow's milk allergy. ${ }^{28}$ Neonatal LGG colonization shows effects on enhancing intestinal functional maturation in mice. ${ }^{11}$ However, information regarding the mechanisms underlying the beneficial effects of probiotics on growth in early life is limited. Therefore, we asked whether p40 supplementation from postnatal day 2 to 21 promoted growth and maturation of the intestine in conventionally raised wild-type mice.

Bodyweight gain is a marker for growth at the early stages of life in most animal species. Compared to mice receiving control hydrogels, mice treated with p40 showed significantly increased bodyweight gain from postnatal day 8 to 20 (Fig. 2a, b). No differences of bodyweight between no-p40 and p40-treated groups were found in mice older than 3-week-old (Fig. 2a, b). These results suggest that neonatal p40 supplementation does not affect bodyweight in adulthood.

Postnatal growth requires functional maturation of the gastrointestinal tract for nutritional supply. Since functional maturation of the intestine occurs at weaning in mice, we investigated the effects of neonatal supplementation of p40 on intestinal epithelial cell proliferation, differentiation, and tight junction formation in 2and 3-week-old mice.

To assess proliferation capacity, we examined Ki67 gene expression levels by real-time PCR analysis and Ki67-expressing
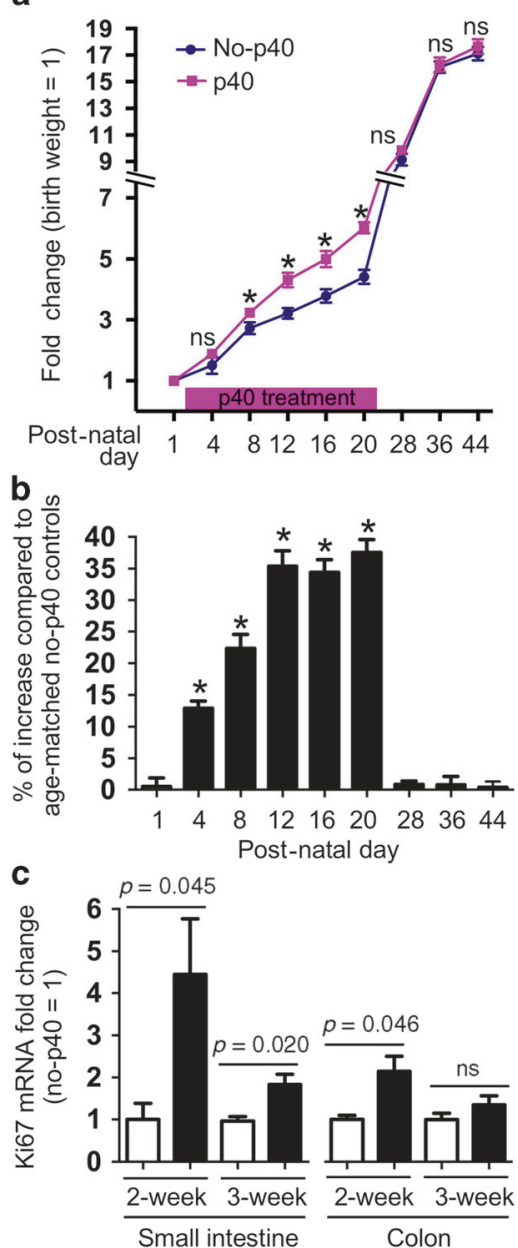

d

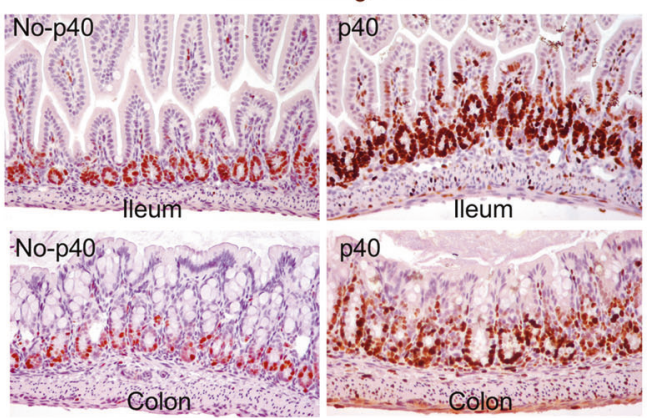

e
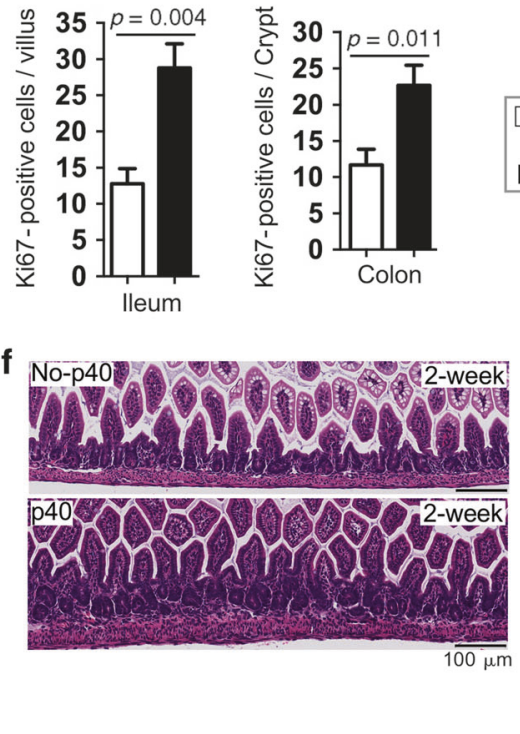

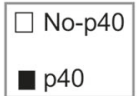

9

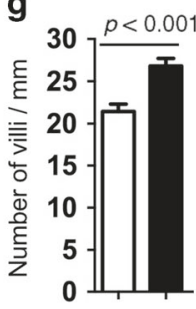

Fig. 2 p40 treatment increases growth and epithelial cell proliferation before weaning in wild-type mice. Mice were treated with p40containing hydrogels at $0.5,1$, and $1.5 \mu \mathrm{g} /$ day at postnatal days $2-6,7-13$, and $14-21$, respectively. As control, pups were treated with hydrogels without p40 (no-p40). a Bodyweight was recorded. The fold change of bodyweight was calculated by comparing the bodyweight at the indicated postnatal day to the bodyweight at birth of the same pup. $\mathbf{b}$ The percentage of increase $=(\mathrm{p} 40$ bodyweight-average of no-p40 bodyweight)/average of no-p40 bodyweight, at the matched postnatal day. c Real-time PCR analysis was performed to detect Ki67 gene expression in the small intestine and the colon. The average of mRNA expression levels in 2- and 3-week-old mice in the no-p40 group were set as $100 \%$, and the mRNA expression level of each mouse was compared to the average at the same age. $\mathbf{d}$, e lleal and colonic tissues from 2-week-old mice were immunostained using an anti-Ki67 antibody and a horseradish peroxidase-conjugated secondary antibody. Slides were developed using DAB and counterstained with hematoxylin. The numbers of positively stained cells are shown. $\mathbf{f}-\mathbf{g}$ lleal tissues from 2 -weekold mice were prepared for H\&E staining and the number of villi per $\mathrm{mm}$ is shown. In $\mathbf{a}, \mathbf{b}$, no-p40 group: $n=15 ; \mathrm{p} 40 \mathrm{group}: n=17$. In $\mathbf{c}, \mathbf{g} n=$ $5-7$ in each group 
cells by immunostaining. p40 treatment significantly increased Ki67 gene expression in the small intestine and the colon of 2week-old mice (Fig. 2c). However, p40 treatment had a diminished effect on increasing Ki67 gene expression in the small intestine and no effect in the colon of 3-week-old mice (Fig. 2c). Consistent with these findings, significant increases of Ki67-expressing cells in the small intestine and the colon of 2week-old mice treated with p40 were observed (Fig. 2d, e). In addition, morphologic evaluation showed that p40-treated mice exhibited increased density of villi in the small intestine of 2week-old (Fig. 2f, g). Cell proliferation in the small intestine and the colon in 7-week-old mice with neonatal p40 treatment was similar to those with no-p40 treatment. The numbers of Ki67positive cells were $16.7 \pm 6.8$ /villus in the ileum and $11.7 \pm 4.8$ / crypt in the colon in mice with no-p40 treatment, and $17.7 \pm 2.8$ / villus and $10.9 \pm 5.8$ /crypt in mice receiving neonatal p40 supplementation ( $p>0.05$ for Ki67-positive cells in the ileum and the colon). These results suggest that the effect of neonatal p40 Supplementary on intestinal epithelial cell proliferation is limited to the early life stages.

During development, intestinal epithelial cells migrate out of the crypt as they terminally differentiate into absorptive and secretory cell types. We examined a digestive enzyme, sucroseisomaltase expression in enterocytes in the small intestine and a secretary protein, MUC2 in Goblet cells in the colon by immunostaining and real-time PCR analysis. As compared to mice without p40 treatment, significant membrane localization of sucrose-isomaltase was found in the 3-week-old mice with p40 treatment (Fig. 3a). p40 treatment also significantly increased sucrose-isomaltase gene expression levels in the small intestine of 2- and 3-week-old mice (Fig. 3b). Furthermore, the numbers of MUC2-positive cells (Fig. 3c, d) and Muc2 gene expression levels (Fig. 3e) in the colon were significantly increased in 3-week-old, but not 2-week-old mice with p40 treatment. These data suggest that promotion of differentiation by $\mathrm{p} 40$ in mice occurs at the weaning time.

Development of the barrier function serves as one of the important events to establish the integrity of the postnatal intestine for maintenance of homeostasis. p40 has been found to preserve barrier function against pro-inflammatory cytokines and hydrogen peroxide-induced disruption of tight junctions in vivo and in vitro. ${ }^{17,29,30}$ Maturation of the epithelial barrier in mice starts with weaning. Thus, we next studied whether p40 enhanced the tight junction formation at the early stage in mice. Claudin 3 (CLDN3) is a tight junctional protein and its expressional level is the highest in 3-week-old mice. ${ }^{31}$ We found that p40 treatment stimulated the levels of Cldn3 mRNA expression in both 2- and 3week-old mice (Fig. 4a). Furthermore, immunostaining of ZO-1 showed that p40 treatment led to increased ZO-1 membrane localization in the small intestinal epithelial cells (Fig. 4b), which is an indication of tight junction formation. These data suggest that p40 promotes tight junction formation in neonatal mice.

Thus, compared to mice receiving hydrogels only and another control, treatment of mice with albumin-containing hydrogels at the same dosage of p40 treatment (Supplementary Figure 1), neonatal p40 treatment increased cell proliferation, differentiation, and tight junction formation in 2- and 3-week-old mice, suggesting that $\mathrm{p} 40$ has the potential to promote intestinal functional maturation.

Neonatal p40 treatment augments IgA production in early life in wild-type mice

Development of the intestinal immune system depends on the colonization of the gut by microbiota. ${ }^{2}$ Germ-free mice exhibit decreased number of $\operatorname{lgA}$-secreting B cells in the intestine, ${ }^{32}$ which can be counteracted by introduction of bacterial flora. ${ }^{33}$ We have found that p40 up-regulated APRIL expression in intestinal epithelial cells, thereby increasing IgA class switching in B cells and $\lg \mathrm{A}$ production in the intestine of adult mice. ${ }^{16}$ Thus, we examined the effects of neonatal p40 treatment on both its $\lg A$ production in the early stages of life. The mRNA levels of April in the small intestine were significantly increased in both 2- and 3week-old mice with p40 treatment (Fig. 5a). The IgA level in feces was measured using enzyme-linked immunosorbent assay (ELISA). The fecal IgA levels in 2- and 3-week-old mice with p40 treatment were significantly higher than those of age-matched non-treated mice (Fig. 5b). Consistent to these data, immunostaining showed that p40 treatment increased the number of IgA-expressing cells in lamina propria of the small intestine in 3-week-old mice (Fig. $5 \mathrm{c}$, d), which suggests that stimulation of IgA production may require accumulative consequence of the p40-stimulated APRIL production.

Transactivation of EGFR in the intestinal epithelial cells mediates the effects of $p 40$ on functional maturation of the intestine in early life

It is well known that activation of EGFR stimulates cell proliferation, differentiation, and survival. ${ }^{19,20}$ EGFR signaling promotes postnatal growth through regulation of intestinal functional maturation. ${ }^{11}$ Since we have found that transactivation of EGFR in the intestinal epithelial cells by p40 mediates protection of intestinal epithelium in colitis ${ }^{17}$ and IgA production, ${ }^{16}$ we next determined the requirement of EGFR in intestinal epithelial cells for p40-regulated intestinal development using transgenic mice with constitutive deletion of Egfr in the intestinal epithelial cells $\left(\right.$ Egfr $^{f l f f}$-Vil-Cre mice). Their littermates, Egfr ${ }^{f / f l}$ mice, were used as control. Based on the identified effects of p40 on proliferation in 2week-old wt pups and differentiation, tight junction formation, and $\lg \mathrm{A}$ production in 3-week-old wt mice, we evaluated these parameters in 2-week and 3-week-old Egfr ${ }^{f / f l}$-Vil-Cre mice, as compared to those in age-matched $\mathrm{Egfr}^{f / f l}$ mice.

p40 promoted bodyweight gain in 2-week-old $\mathrm{Egfr}^{\mathrm{fl} / \mathrm{fl}}$, but not Egff ${ }^{f / f f}$-Vil-Cre mice (Fig. 6a, b). The proliferative effects of p40 in 2week-old pups, detected by Ki67 gene expression and the number of Ki67-positive cells, and the density of villi in the small intestine were observed in $\mathrm{Egfr}^{f / / f l}$ pups; however, p40 failed to stimulate proliferation in $\mathrm{Egfr}^{\mathrm{fl} / \mathrm{fl}}$-Vil-Cre mice (Fig. 6c, Supplementary Figure 2a-d). Furthermore, p40 treatment had no effects on cell differentiation detected by expression of sucrase-isomaltrase (Fig. 6d, e), tight junction formation by ZO-1 staining (Fig. 6f), fecal IgA levels (Fig. 6g), and expression of April (Fig. 6h) in Egfr f/ffl Vil-Cre mice. These results indicate that transactivation of EGFR in intestinal epithelial cells mediates the effects of p40 on cell proliferation, differentiation, tight junction formation, and $\lg \mathrm{A}$ production during development in early life.

Neonatal p40 treatment has long-term outcomes for prevention of colitis in adult mice

To understand long-term outcomes of administering probiotics in early life, we demonstrated a preventive effect of neonatal LGG colonization on colitis in adulthood. ${ }^{11}$ Therefore, we asked if neonatal p40 treatment was able to prevent intestinal inflammation in adult mice. Two mouse models of colitis were used in 6- to 7-week-old mice. 2,4,6-trinitrobenzenesulfonic acid (TNBS)induced colitis is mediated by interleukin-12 (IL-12) driven Th1 immune responses, including increased tumor necrosis factor (TNF) and interferon- $\gamma$ (IFN- $\gamma$ ) production. ${ }^{34}$ The dextran sulfate sodium (DSS) mouse model of acute colitis is well-characterized by increased intestinal epithelial injury and production of inflammatory cytokines. ${ }^{35}$

TNBS induced histological changes, including disruption of the epithelial monolayer and inflammatory cell infiltration with an inflammation score of $2.8 \pm 0.61$ in mice without neonatal p40 treatment. No inflammation was induced by TNBS in mice with neonatal p40 supplementation (score: 0 in all mice in this group, $p$ $<0.05$ ) (Fig. 7a, b). TNBS up-regulated levels of TNF and IFN- $\gamma$ 
a
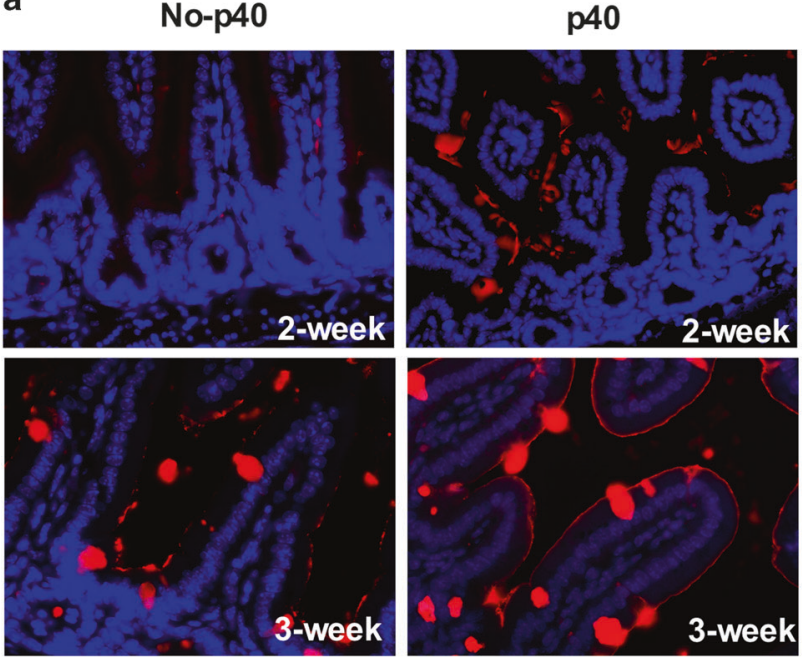

Sucrase-isomaltase

C
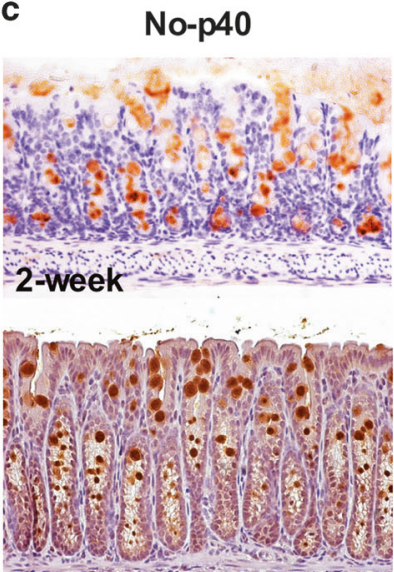

3.week
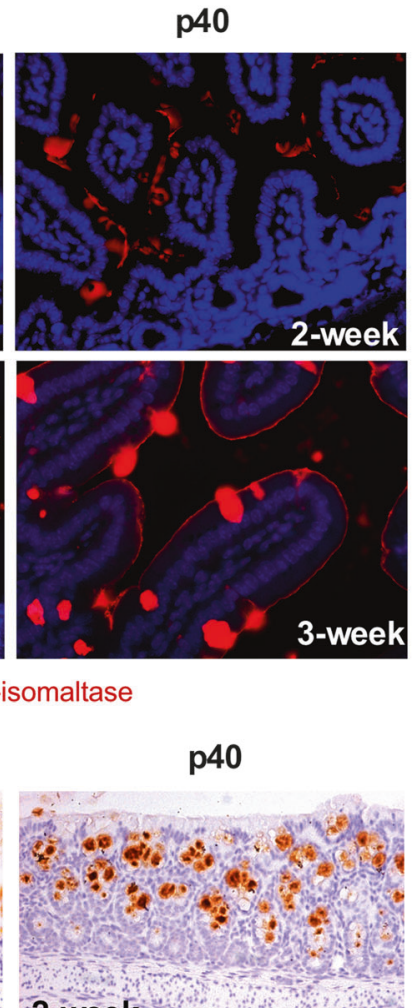

2-week

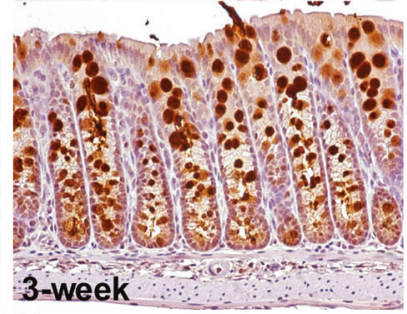

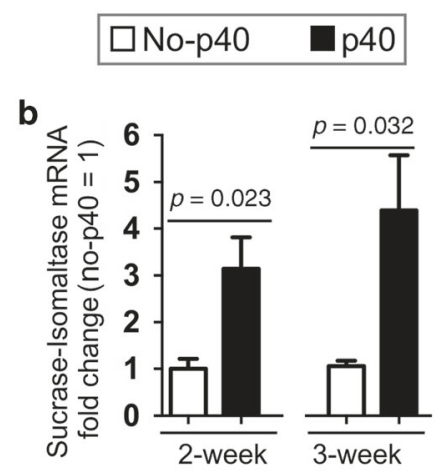

d

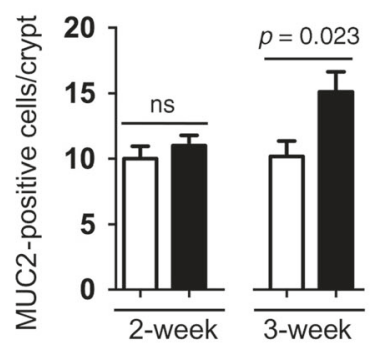

e

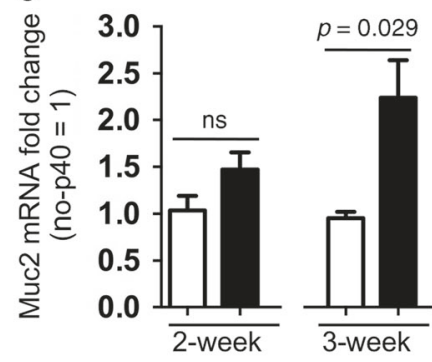

Fig. 3 p40 promotes intestinal cell differentiation before weaning in wild-type mice. Mice were treated with p40 as described in Fig. 2. Fixed tissue sections and RNA were prepared from the small intestine and the colon of 2- and 3-week-old mice. a The small intestinal tissue sections were immunostained using an anti-Sucrase-isomaltase antibody and a Cy3-labeled secondary antibody (red staining). Nuclei were stained with DAPI (blue staining). b, e Real-time PCR analysis was performed to detect gene expression levels of Sucrase-isomaltase in the small intestine and Muc2 in the colon. The average of mRNA expression levels in 2- and 3-week-old mice in the no-p40 group was set as $100 \%$, and the mRNA expression level of each mouse in p40-treated group was compared to the average of the same age group. $\mathbf{c}$, $\mathbf{d}$ The colon tissue sections were immunostained using an anti-Muc2 antibody and a horseradish peroxidase-conjugated secondary antibody (brown staining) and were developed using DAB. Sections were counterstained with hematoxylin. The numbers of MUC2 positively stained cells are shown. $n$ $=5-7$ mice in each group. Images in a and c represent 3-5 mice in each group

expression in the colonic mucosa in mice without p40 treatment, which was blocked in mice with neonatal p40 supplementation $(p$ $<0.05$, Fig. 7c).

DSS-treated mice exhibited injury and acute colitis with massive colon ulceration, crypt damage, and severe inflammation. These abnormalities were reduced in mice with neonatal p40 treatment (Fig. 7d). In the no-p40 group, DSS treatment resulted in an injury/ inflammation score of $9.857 \pm 0.654$, which was significantly decreased in the neonatal p40-treated group $(7.857 \pm 0.581, p<$ 0.05 , Fig. 7e). DSS induces neutrophil infiltration in the colon leading to increasing colonic MPO activity, which is therefore an inflammatory marker for colitis. Neonatal p40 treatment reduced DSS-increased MPO activity in the colon (Fig. 7f). Furthermore, DSS treatment significantly increased TNF, KC, and IL-6 mRNA levels in the no-p40 group, which were reduced in mice with neonatal p40 treatment (Fig. 7g).

These data suggest that neonatal p40 treatment plays roles in prevention of intestinal inflammation in adulthood.
Neonatal p40 treatment has persistent effects on promoting protective immune responses in adult mice

We next studied whether neonatal p40 treatment had persistent effects on modulating protective immune responses in adulthood. We found that the fecal $\lg A$ levels were higher in adult mice receiving neonatal p40 treatment, as compared to those in the nop40 group (Fig. 8a, b). Neither TNBS nor DSS affected the level of fecal IgA in the no-p40 group (Fig. 8a, b). The fecal IgA levels were maintained higher in both TNBS- and DSS-treated mice receiving neonatal p40 supplementation (Fig. 8a, b). Further, in mice receiving neonatal p40 supplementation, the IgA level in TNBStreated mice was higher than that in the ethanol-treated group (Fig. 8a). Since intestinal IgA plays roles in maintenance of homeostasis, $^{36}$ the persistent increase of $\operatorname{lgA}$ production by neonatal p40 supplementation might contribute to enhancing the defense capacity against inflammation in adult mice.

The mucus layer of the gastrointestinal tract serves as the first line of intestinal defense against injury and infection. ${ }^{37}$ Although 


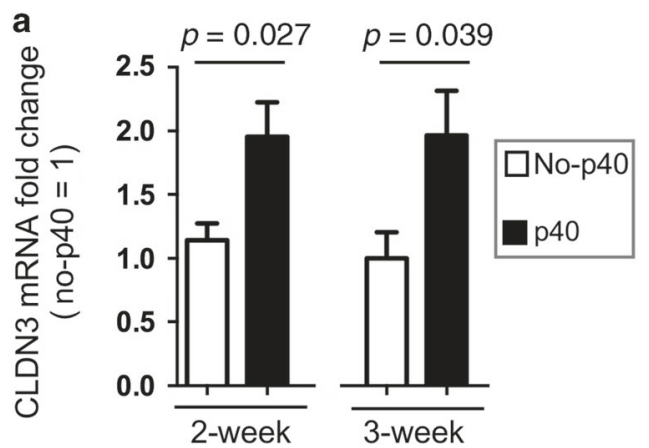

b
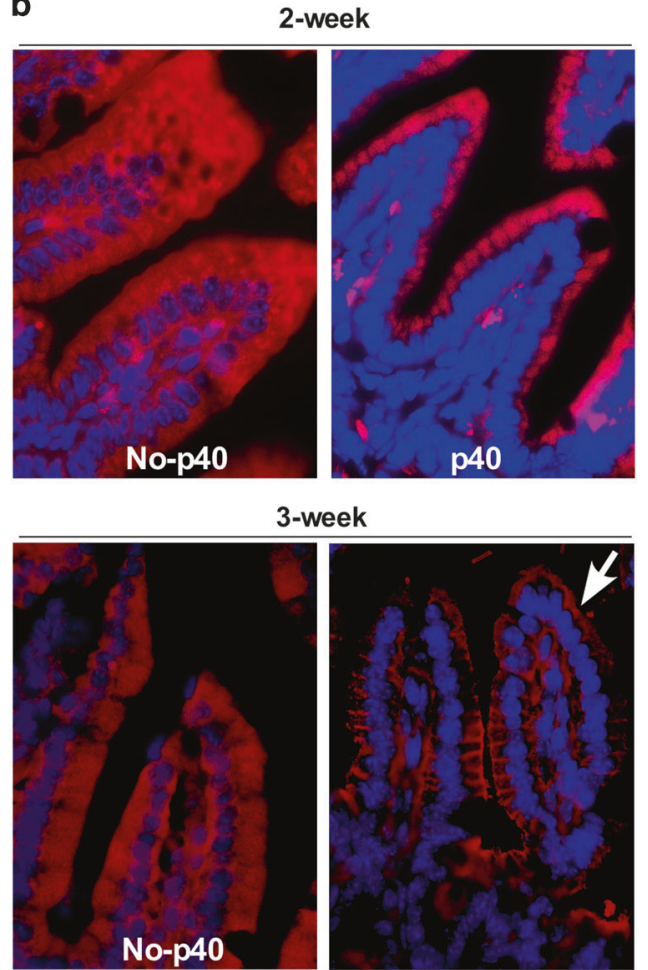

Red: ZO-1 Blue: DAPI

Fig. 4 p40 enhances tight junction formation in the small intestine of wild-type mice before weaning. Mice were treated with p40 as described in Fig. 2. Fixed tissue sections and RNA were prepared from the small intestine of 2- and 3-week-old mice. a Real-time PCR analysis was performed to detect CLND-3 expression. The average of mRNA expression levels in 2- and 3-week-old mice in the no-p40 group was set as $100 \%$, and the mRNA expression level of each mouse in p40-treated group was compared to the average of the same age group. $n=5-7$ in each group. b Tissue sections were immunostained using an anti-ZO-1 antibody (red staining) and a Cy3-labeled secondary antibody (red staining). Nuclei were stained with DAPI (blue staining). Images represent five mice in each group

neonatal p40 treatment increased MUC2 production before weaning (Fig. $3 \mathrm{C}-\mathrm{e}$ ), it did not affect the numbers of MUC2positive cells (Fig. 8c, d, Supplementary Figure 3a) and Muc2 gene expression in the colon (Fig. 8e, Supplementary Figure $3 \mathrm{~b}$ ) in adult mice. There were no changes in Muc2 gene expression and the numbers of MUC2-positive cells in the colon of mice with TNBS treatment (Supplementary Figure 3a, b). DSS treatment reduced Muc2 gene expression and the numbers of MUC2-positive cells in the colon in no-p40-treated groups, which was prevented in mice with neonatal p40 treatment (Fig. 8c-e). Preserving mucin production in DSS-treated mice with neonatal p40 treatment may be associated with less injury and colitis in these mice. In return, maintaining the mucin level might contribute to protecting intestinal epithelium again injury and inflammation in DSS-treated mice with neonatal p40 supplementation.

The gut microbiota contribute to induction and maintenance of intestinal regulatory $T$ cells (Tregs) expressing the transcription factor forkhead box P3 (Foxp3), which play roles in the maintenance of the mucosal barrier homeostasis. ${ }^{38}$ Therefore, we evaluated the long-term effects of neonatal p40 treatment on differentiation of Tregs in the intestine and the mechanism underlying this effect. Flow cytometry analysis was performed to examine populations of $\mathrm{CD} 4^{+}$Foxp $3^{+} \mathrm{CD} 25^{+}$cells in the lamina propria of the small intestine and colon and in Peyer's Patches. Neonatal p40 treatment did not affect the population of the CD4 ${ }^{+}$ cells in the lamina propria of the small intestine and the colon and in Peyer's patches in adult mice (Supplementary Figure 4). Neonatal p40 treatment significantly increased the percentages of $\mathrm{CD}^{+}{ }^{+} \mathrm{Foxp}^{+} \mathrm{CD}^{2} 5^{+}$cells in the lamina propria of the small intestine and the colon (Fig. 9a, b). Furthermore, TNBS treatment decreased the percentage of $\mathrm{CD} 4{ }^{+} \mathrm{Foxp} 3^{+} \mathrm{CD} 25^{+}$cells in the lamina propria of the small intestine and the colon in no-p40 groups, which were up-regulated in mice with neonatal p40 treatment (Fig. 9b). However, there was no significant effect of neonatal p40 treatment on induction of Tregs differentiation in Peyer's Patches (Fig. 9a, b). TNBS treatment did not alter the percentage of $\mathrm{CD} 4^{+}{ }^{+}$oxp $3^{+} \mathrm{CD} 25^{+}$cells in Peyer's patches (Fig. 9b). Thus, these data suggest that neonatal p40 treatment enhances induction of Tregs in the lamina propria of the small intestine and the colon, which may contribute to prevention of TNBS-induced colitis in adult mice.

\section{DISCUSSION}

Supplementation of probiotics in early life as a strategy for benefiting growth and preventing diseases has attracted increasing clinical and research interest. For examples, probiotics have shown preventive and/or treatment effects on infectious diarrhea in infants and young children ${ }^{8,9}$ and antibiotic-associated diarrhea in children. ${ }^{9}$ Therefore, understanding of the potential roles and mechanisms underlying the influence of probiotics on the gastrointestinal tract at the early developmental stage, which is characterized by immature function and immunity, is important. Results from this study support the important developmental effects of p40 on the intestinal tract, including significantly promotion of epithelial cell proliferation and differentiation in 2and 3-week-old mice, respectively, and contribution to epithelial cell tight junction formation and IgA production starting at 2week-old. Interestingly, neonatal p40 treatment had long-term positive outcomes on IgA production and prevention of colitis, but did not affect bodyweight in adulthood. Furthermore, we have also examined the effect of p40 on intestinal stem cells during development. Our data showed that there was no difference of the number of cells expressing Lgr5 in the ileum and the colon in p40-treated 2-week-old mice, as compared to that in the control group, which suggest that p40 may not affect stem cell proliferation (Supplementary Figure 5). These results provide useful insight into the roles of probiotic-derived factors in maturation of intestinal functions and immunity. Furthermore, since concerns regarding the utility of viable probiotics have been raised, including the challenges of ensuring the bioavailability and efficacy of probiotic bacteria in vivo as well as biosafety issues, such as bacterium-associated infections ${ }^{39}$ and sepsis ${ }^{40}$ in very young and immunocompromised patients, applying probioticderived factors may serve as a safe and efficacious surrogate to the use of probiotics.

Findings from this study indicate the involvement of EGFR signaling in p40-regulated proliferation, differentiation, and tight junction formation in intestinal epithelial cells during development and persistent $\lg \mathrm{A}$ production. $\mathrm{p} 40$ has been 
a

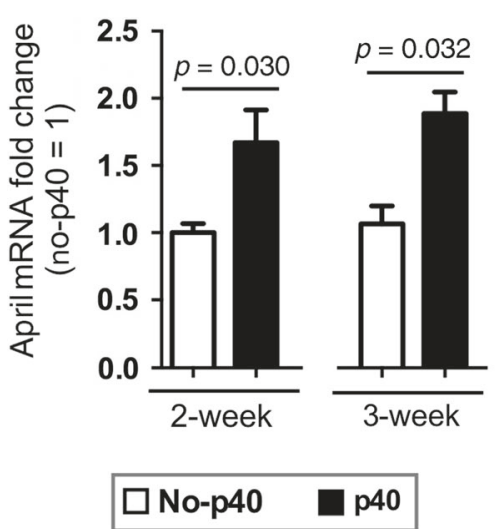

b

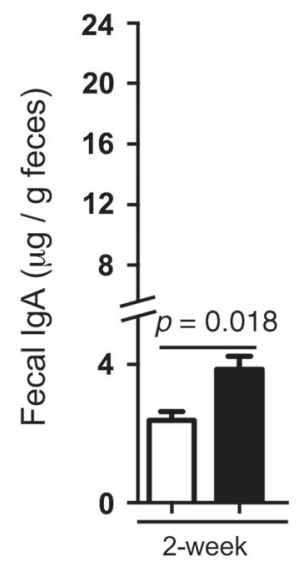

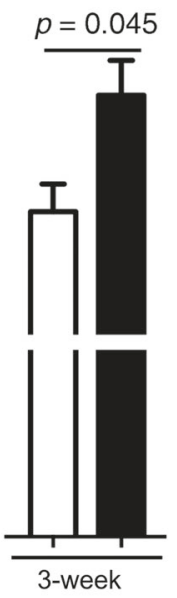

C

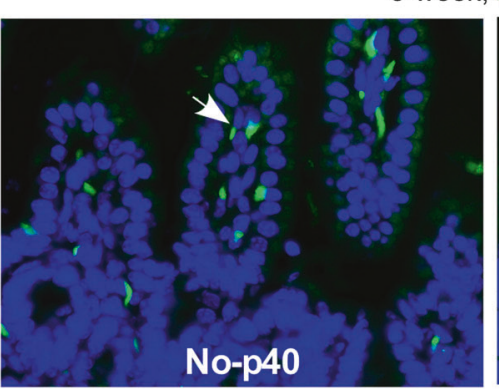

3-week, IgADAPI

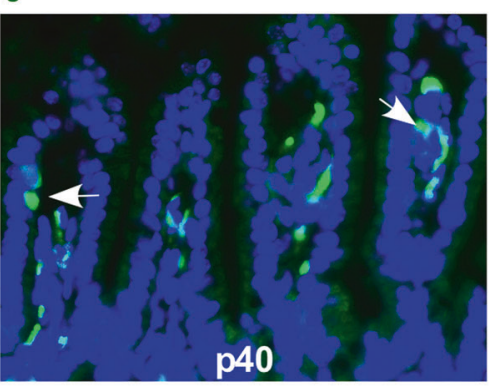

d

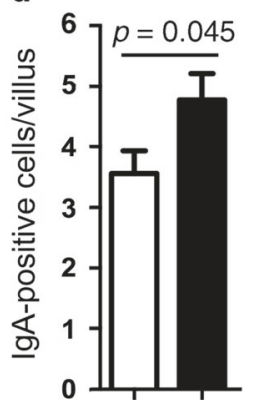

Fig. 5 p40 increases production of IgA in 3-week-old wild-type mice. Mice were treated with p40 as described in Fig. 2. a RNA isolated from small intestinal tissues was used for real-time PCR analysis of April expression. The average of mRNA expression levels in 2- and 3-week-old mice in the no-p40 group was set as $100 \%$, and the mRNA expression level of each mouse in the p40-treated group was compared to the average of the same age group. $n=5-7$ mice in each group. b Feces were collected at the indicated ages. Fecal slgA levels were determined using ELISA assay. $n=3-5$ in the no-p40 group, $N=7$ in the p40 group. $\mathbf{c}$, $\mathbf{d}$ Tissue sections were prepared for immunostaining of IgA. Nuclei were stained with DAPI (blue staining). Green arrows indicate IgA positively stained cells in the lamina propria. The number of IgA-positive cells per villus is shown. $n=5-9$ in each group. Images represent five mice in each group

shown to stimulate HB-EGF release for transactivation of EGFR in intestinal epithelial cells. ${ }^{18}$ Previous results strongly support the involvement of transactivation of EGFR by 440 for preserving intestinal epithelial cell barrier function upon injury ${ }^{14,17,29}$ and up-regulation of April expression in intestinal epithelial cells for IgA production in adult mice. ${ }^{16}$ Results from this study broaden our knowledge of the effects of p40-activated EGFR on intestinal epithelial cells into the developmental stage by demonstrating promotion of tight junction formation and $\lg A$ production. Regarding growth and epithelial cell proliferation, it is well known that EGFR ligands from maternal and endogenous sources play roles in postnatal growth and intestinal epithelial cell proliferation. ${ }^{41}$ This evidence is consistent with our finding that p40 stimulates epithelial cell proliferation through EGFR transactivation. However, it has been reported that knockdown of triple ligands, EGF, amphiregulin, and transforming growth factor-a (TGFa), causes growth retardation and defects in intestinal development, but not production of digestive enzyme. ${ }^{41}$ Although we found that p40 failed to up-regulate digestive enzyme production in mice with deletion of EGFR in epithelial cells, it is possible that other signaling pathways regulated by $\mathrm{p} 40$ mediate the effects of p40 on differentiation. These pathways may have interactions with EGFR signaling for their regulatory effects on differentiation. It should be noted that overexpression of EGFR ligands has been shown to reduce muscle, fat, and bone proportions. ${ }^{42}$ This notion should be taken into account for using any approaches related to upregulation of EGFR ligands, including p40, to promote growth in early life.
Increasing evidence supports the long-term health outcomes from the acquisition of the gut microbiota in early life. Studies have revealed the presence of a window of opportunity in early life for colonization of microbiota which shape the host immune functions in adulthood. ${ }^{43}$ In addition, altering the intestinal microbiota during development results in a disturbed colonic microbiome and metabolic consequences in adult mice. ${ }^{44}$ Two long-term effects of neonatal p40 treatment in adult mice were identified in this study, enhanced IgA production and decreased susceptibility to colitis. It is well known that $\lg A$ in the intestinal lumen plays an important role in maintaining mucosal homoeostasis and protecting the host against pathogenic infections. ${ }^{36}$ The roles of IgA in early life was further demonstrated that early exposure to maternal secretory $\lg A$ prevented DSS-induced colonic damage in adult mice, which was associated with upregulation of expression of gens involved in cell metabolism and repair intestinal epithelial cells. ${ }^{45}$ Thus, this evidence supports the roles of increased IgA production by neonatal p40 treatment in prevention of colitis in adults.

One interesting finding from this study is that neonatal p40 treatment has more potent effects on prevention of TNBS-induced colitis, as compared to that on DSS-induce intestinal injury and colitis. In addition, neonatal p40 treatment completely blocked TNBS-induced TNF and IFN- $\gamma$ production in adult mice. However, DSS-induced proinflammatory cytokine production was decreased, but not completely inhibited in mice with neonatal p40 treatment. This evidence may be related to the further increase of IgA production in TNBS-, but not in DSS-colitis in mice with neonatal p40 treatment. Furthermore, the long-term effect of 
a
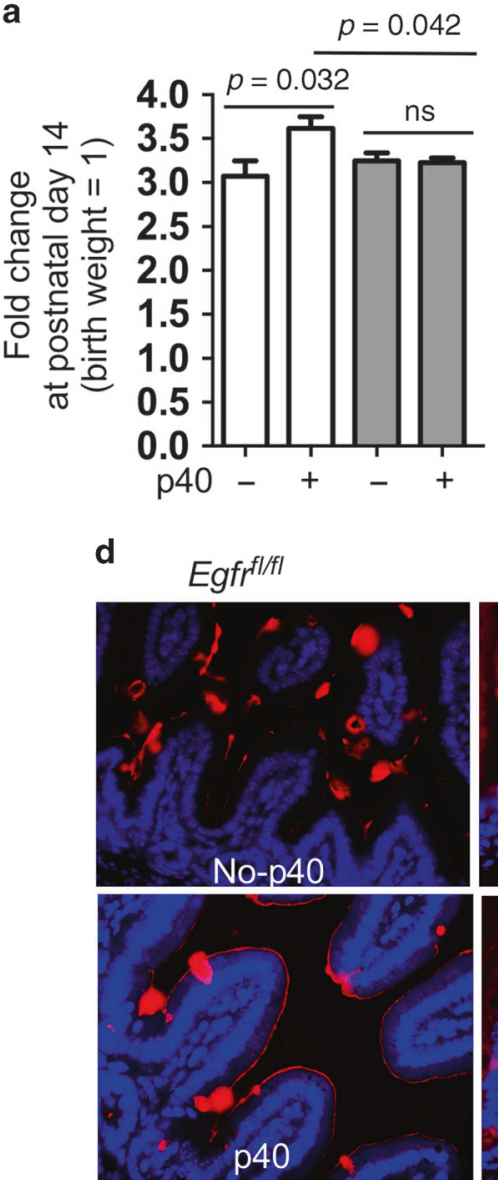

b

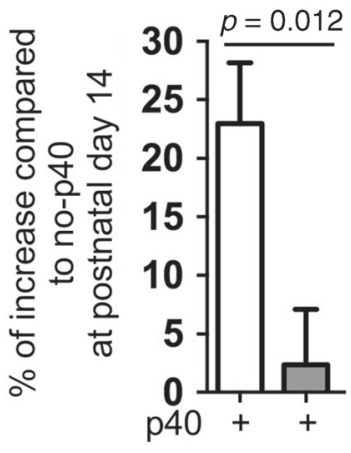

\section{Egfrllffl-Vil-Cre}
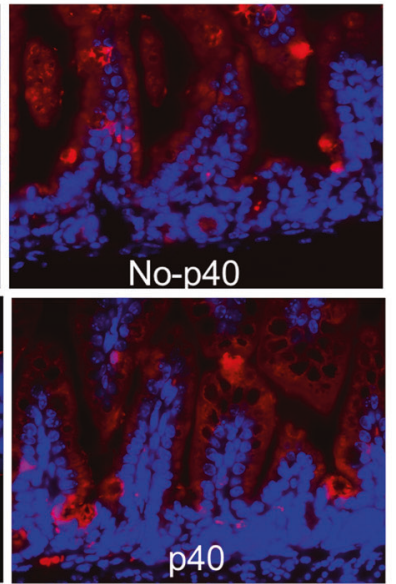

Sucrase-isomaltase, 3 week
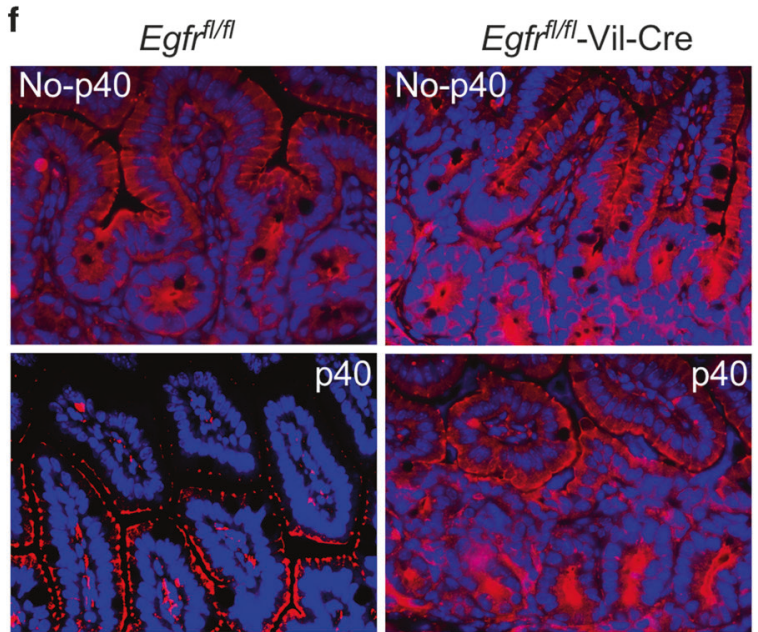

g
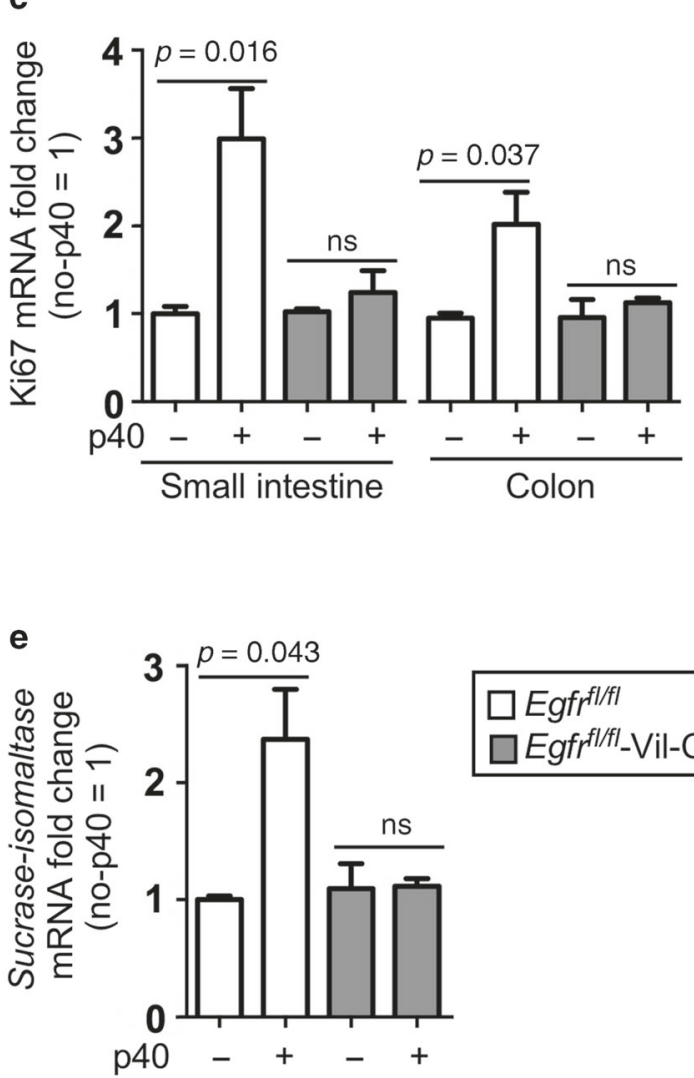

ZO-1, 3 week

Fig. 6 EGFR expression in intestinal epithelial cells mediates developmental effects of p40 in pups. Egfr $\mathrm{fr}^{\mathrm{f} / f l}$ and Egfr $\mathrm{Alf}^{\mathrm{f} / \mathrm{f}}$-Vil-Cre pups were treated with p40 as described in Fig. 2. a The fold change of bodyweight (a) and the percentage of bodyweight increase (b) at postnatal day 14 were calculated as described in Fig. 2. c Real-time PCR analysis was performed to detect Ki67 gene expression in the small intestine and the colon of 2-week-old mice. d, e The ileum tissues from 3-week-old mice were prepared for sucrose-isomaltase immunostaining and real-time PCR analysis to detect gene expression. $\mathbf{f}$ The colonic tissues from 3-week-old mice were prepared for ZO-1 immunostaining. $\mathbf{g}$ Feces from 2-weekold mice were collected for ELISA to detect fecal IgA levels. $\mathbf{h}$ Real-time PCR analysis was performed to detect APRIL gene expression in the small intestine of 2-week-old mice. In $\mathbf{c}, \mathbf{h}$ the average of mRNA expression levels in the no-p40 group was set as $100 \%$, and the mRNA expression level of each mouse was compared to the average. In a-c, e, and $\mathbf{f}-\mathbf{h}, n=5-9$ in each group. In d, f, no-p40: $n=3-5 ; \mathbf{p} 40: n=5-7$ in each group 

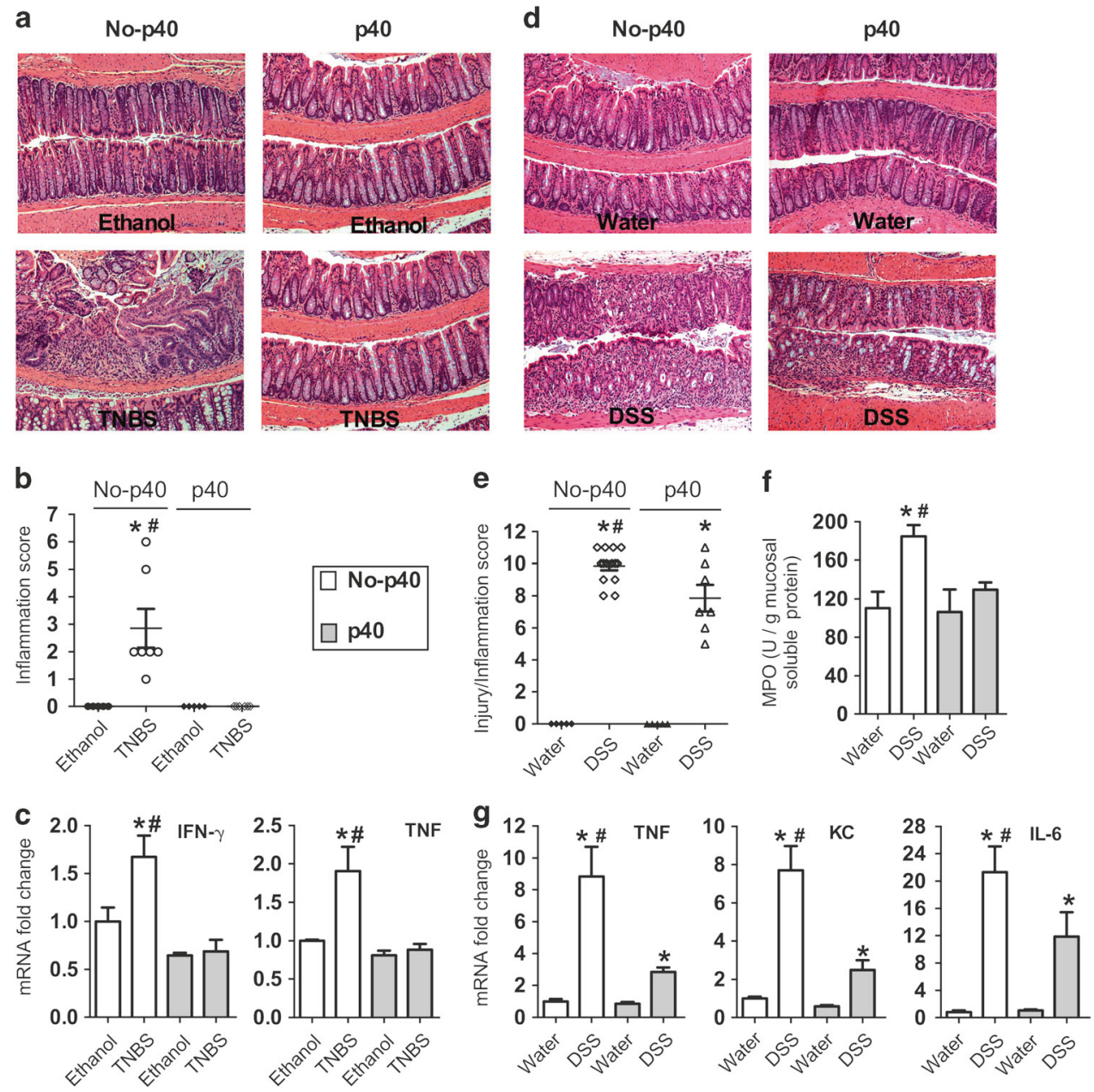

Fig. 7 Neonatal p40 treatment prevents colitis in adult mice. Mice were treated with p40 from postnatal day 2 to day 21 , as described in Fig. 2 . Colitis was induced in 7-week-old mice by TNBS in ethanol intrarectally (a-c) and by $3 \%$ DSS in drinking water for 4 days (d-f). Mice receiving ethanol and water were used as controls for TNBS and DSS treatment, respectively. Mice were euthanized 4 days after TNBS treatment and at the fourth day of DSS treatment. $\mathbf{a}, \mathbf{b}$ and $\mathbf{d}$, e Colon sections were stained with H\&E for light microscopic assessment of inflammation. The inflammation/injury scores are shown. $\mathbf{c}, \mathbf{g}$ mRNA was isolated from the colonic tissues for real-time PCR analysis of indicated cytokine mRNA expression levels. The average of cytokine mRNA expression levels in the control mice of the no-p40 group was set as $100 \%$, and the mRNA expression level of each mouse was compared to this average. $\mathbf{f}$ MPO activity in the colonic tissue lysates was detected. ${ }^{*} p<0.05$ compared to the control mice in the no-p40 group. \#p<0.05 compared to the p40 group with TNBS or DSS treatment. $n=5$ in control groups, $n=7$ in TNBS- and DSS-treated groups

neonatal p40 treatment on increasing Tregs differentiation may contribute to shaping the functions of the protective immune responses against TNBS-induced colitis in adulthood.

TGF $\beta$ is a known cytokine to trigger differentiation of Tregs. ${ }^{46}$ We found the effects of $\mathrm{p} 40$ on TGF $\beta$ production by young adult mouse colonic (YAMC) epithelial and mouse small intestinal epithelial (MSIE) cell lines. The p40 treatment stimulated release of TGF $\beta$ in cell culture medium and up-regulated TGF $\beta$ gene expression in YAMC and MSIE cells in a time-dependent manner (Supplementary Figure $6 a, b)$. Furthermore, the effects of p40-conditioned medium from YAMC and MSIE cells on differentiation of $C D 4^{+} T$ cells isolated from the mouse spleen were tested in the presence and absence of a TGF $\beta$ neutralizing antibody. CD4 ${ }^{+}$T cells were also treated with p40 to examine whether p40 had direct effects on differentiation of $\mathrm{T}$ cells. Compared to the conditioned medium from control cells, p40-conditioned medium significantly increased the percentage of $\mathrm{CD}^{+}{ }^{+} \mathrm{Foxp}^{+}$cells, which was blocked by the TGF $\beta$ neutralizing antibody. Direct p40 treatment did not affect the percentage of $\mathrm{CD}^{+}{ }^{+} \mathrm{Foxp}^{+}$cells in $\mathrm{CD}^{+} \mathrm{T}$ cell culture (Supplementary Figure $6 \mathrm{c}$, d). Thus, TGF $\beta$ production by intestinal epithelial cells might mediate differentiation of Tregs.
There data also provide information to explain our finding that neonatal p40 supplementation led to induction of Tregs in the lamina propria of the small intestine and the colon, but not in Peyer's patches. Increased TGF $\beta$ level might occur in the lamina propria, but not in Peyer's patches, for promoting differentiation of Tregs in mice with p40 treatment. This explanation is further supported by the result that expression levels of TGF $\beta$ gene were increased in the small intestinal and colonic mucosa of mice with neonatal p40 treatment (Supplementary Figure 7). Therefore, p40 up-regulated TGF $\beta$ production in intestinal epithelial cells may contribute to promoting differentiation of Tregs.

Although probiotics are recommended for use for several diseases, such as infectious diarrhea and antibiotic-associated diarrhea in children, ${ }^{47,48}$ it should be noted that the effectiveness of probiotics on diseases at different ages might vary. ${ }^{49,50}$ Thus, due to the characteristics of each developmental stage, more studies are needed to determine the clinical efficacy of p40 and probiotic-derived factors, especially for age-dependent effects, and duration of treatment.

In summary, by using neonatal treatment of p40 in mice as a model to study the mechanisms of probiotic action in intestinal 


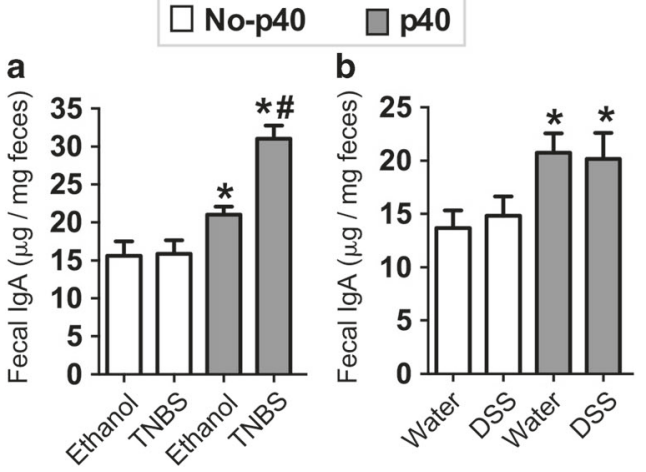

C

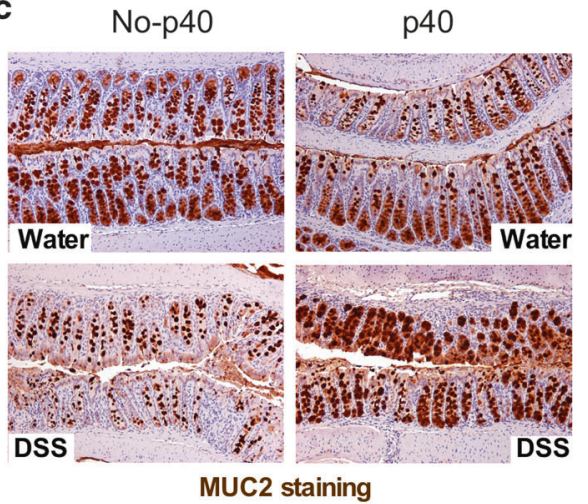

d
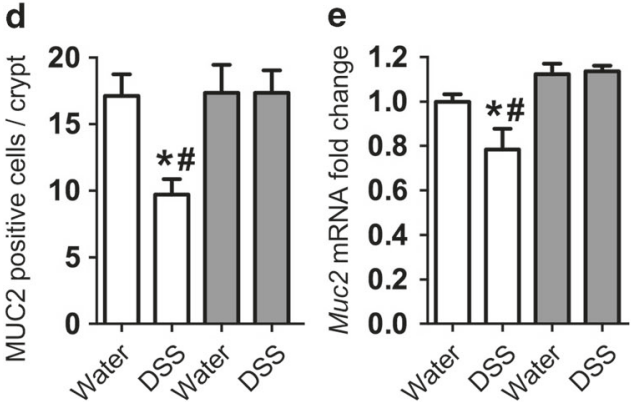

Fig. 8 Neonatal p40 treatment augments innate immunity in adult mice. Mice were treated with $\mathrm{p} 40$ from postnatal day 2 to day 21 , as described in Fig. 2. Colitis was induced in 6- to 7-week-old mice by TNBS and DSS, as described in Fig. 7. a, b The fecal lgA levels were examined using ELISA. ${ }^{*} p<0.01$ compared to the no-p40 group with and without colitis. \#p $<0.05$ compared to the p40 group without TNBS treatment. c, d The colonic tissue sections were immunostained with MUC2 and counterstained with hematoxylin. The numbers of MUC2 positively stained cells are shown. e mRNA was isolated from the colonic tissues for real-time PCR analysis of Muc2 mRNA expression levels. The average of mRNA expression levels in the control mice in the no-p40 group was set as $100 \%$, and the mRNA expression level of each mouse was compared to this average. $n=5-7$ in each group. In $\mathbf{d}, \mathbf{e},{ }^{*} p<0.05$ compared to control mice in the no-p40 group. ${ }^{\#} p<0.05$ compared to the p40 group with DSS treatment

development, this study defines a mechanism for regulating maturation of intestinal functions and innate immunity by $\mathrm{p} 40$ through EGFR signaling in intestinal epithelial cells. Furthermore, identification of neonatal p40 supplementation-induced longterm effects on promoting protective immune responses and preventing against intestinal inflammation in adulthood broadens the potential applications of probiotic-derived factors for health outcomes.

\section{METHODS}

p40 isolation and preparation of p40-containing hydrogels LGG (American Type Culture Collection 53103) was cultured in Lactobacillus MRS broth. As previously described, ${ }^{14}$ p40 was purified from LGG culture supernatant using UNOsphere $S$ ion exchange media (Bio-Rad Laboratories, Hercules, CA) and eluted using Tris buffer $(30 \mathrm{mmol} / \mathrm{l}, \mathrm{pH}$ 7.3) containing sequential concentrations of $\mathrm{NaCl}(100-800 \mathrm{mM})$. Proteins in the eluted fractions were examined by separating in sodium dodecyl sulfate polyacrylamide gel electrophoresis and staining using Colloidal Blue Staining Kit and for western blot analysis using an anti-p40 antibody. ${ }^{14}$ p40 was eluted in the fraction containing $300-500 \mathrm{mM}$ of $\mathrm{NaCl}$. The p40-containing fractions were concentrated using two centrifugal filter devices with molecular weight cutoff of 30 and $50 \mathrm{kDa}$ (EMD Millipore Corporation, Billrica, MA). p40 concentration was determined using a BCA protein assay kit (Pierce Thermo Scientific). Purified p40 was saved at $-80^{\circ} \mathrm{C}$.

The level of endotoxin in p40 isolates was detected using Pierce $^{\mathrm{TM}}$ LAL Chromogenic Endotoxin Quantitation Kit (Pierce Thermo Scientific). The sensitivity tested by this kit is $0.1 \mathrm{EU}$ endotoxin $/ \mathrm{ml}$. The amount of endotoxin in p40 isolates was $<0.03$ $\mathrm{EU} / \mu \mathrm{g}$ p40 protein.

The pectin/zein hydrogels were prepared at room temperature according to the previously published method. ${ }^{17}$ p40 was dissolved in pectin solution $(2.0 \% \mathrm{w} / \mathrm{v})$ in water. Zein solution $(1.0 \% \mathrm{w} / \mathrm{v})$ was prepared in $75 \%$ ethanol solution containing $0.5 \%$ $(\mathrm{w} / \mathrm{v})$ of $\mathrm{CaCl}_{2}$. Then, the p40-containing pectin solution was dropped into the zein solution through a $27 \mathrm{G}$ needle connected to a syringe. After hydrogels ( $4 \mu \mathrm{l} / \mathrm{drop})$ became solid in the zein solution and formed round sheets, hydrogels were washed with water, air-dried, and stored at $4{ }^{\circ} \mathrm{C}$. Two concentrations of p40containing hydrogels were prepared, 0.5 and $1.0 \mu \mathrm{g}$ of $\mathrm{p} 40 /$ hydrogel. As negative controls, pectin/zein hydrogels without $\mathrm{p} 40$ were prepared.

p40 treatment for mice

All animal experiments were performed according to protocols approved by the Institutional Animal Care and Use Committee at Vanderbilt University Medical Center. This study used wild-type C57BL/6, Egfr ${ }^{f l / f}$-Vil-Cre mice on a C57BL/6 background with a constitutive deletion of EGFR in intestinal epithelial cells, ${ }^{17}$ and their littermates, $\mathrm{Egfr}^{f / / f l}$ mice, as controls. Based on statistical analysis for comparing growth rate, gene expression levels of Ki67, sucrose-isomaitase, April, and fecal lgA level in 2-week-old Egfr ${ }^{f / f}$ and $E g f^{f l / f}$-Vil Cre mice without p40 treatment, no differences of these parameters in these two mouse models were identified (Fig. 6). These results suggest that Egfr ${ }^{f / f}$-Vil-Cre mice do not have any defect in the intestinal cell proliferation and differentiation, and IgA production under normal condition.

For each experiment, two female mice from the same litter and one male mouse were housed in the same cage until one to 3 days before delivery. Pups in one litter were treated with p40containing hydrogels at $0.5 \mu \mathrm{g} /$ day (postnatal days $2-6$ ), $1 \mu \mathrm{g} /$ day (postnatal days 7-13), and $1.5 \mu \mathrm{g} /$ day (postnatal days 14-21). As control, pups from the other litter were treated with hydrogels without $\mathrm{p} 40$. Pups received hydrogels through oral administration. Experiments were repeated for at least three times.

Bodyweight was recorded. Percentage of change compared to the bodyweight at birth and the percentage of increase ( $p 40$ bodyweight-average of no-p40 bodyweight)/average of no-p40 bodyweight, at the matched postnatal day) were used to evaluate the growth rate. Intestinal tissues from 2-, 3-, and older than 6-8week-old mice were isolated for preparation of paraffinembeddedtissue sections for immunohistochemistry and hematoxylin and eosin (H\&E) staining. H\&E stained sections were scanned using a Leica SCN400 slide scanner and the number of villi in 2-week-old were counted in at least $1 \mathrm{~cm}$ of the ileum. The average length of villi in the ileum of 2-week-old mice with and 
a

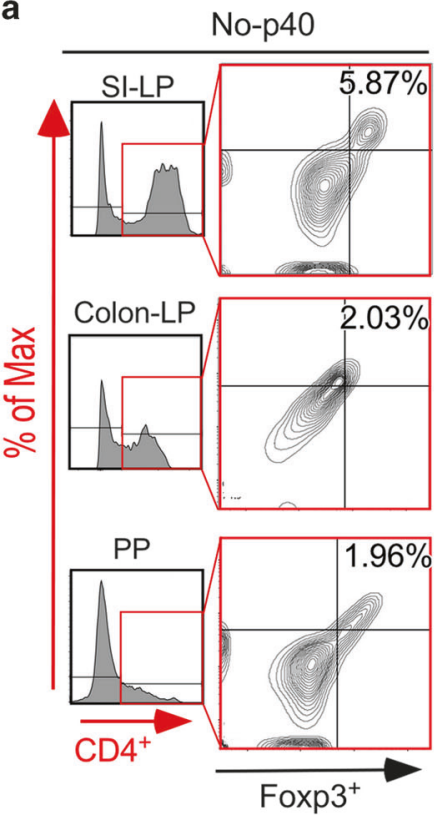

b

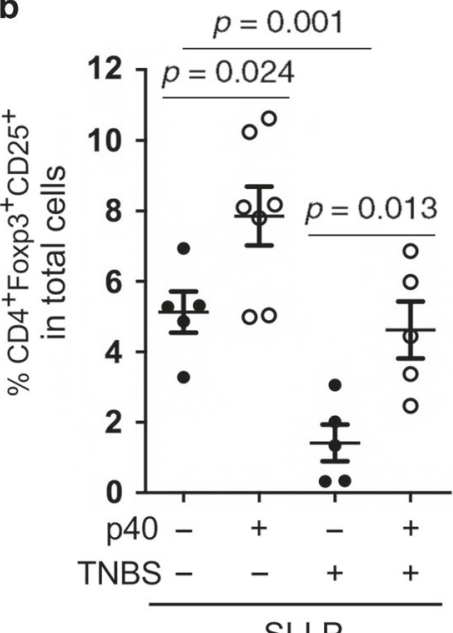

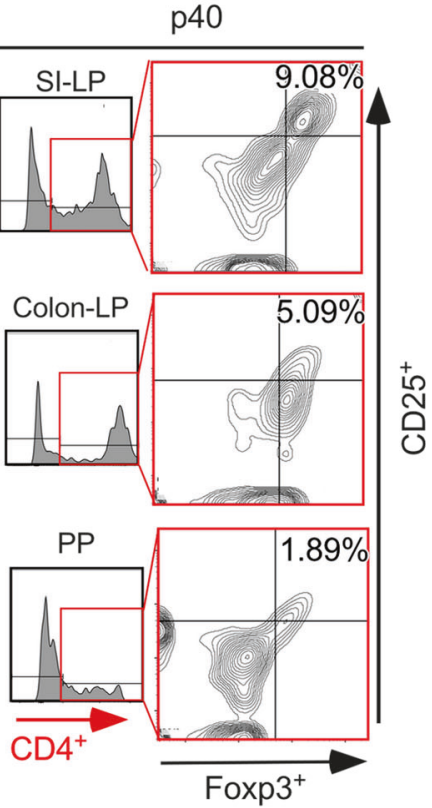

Foxp3
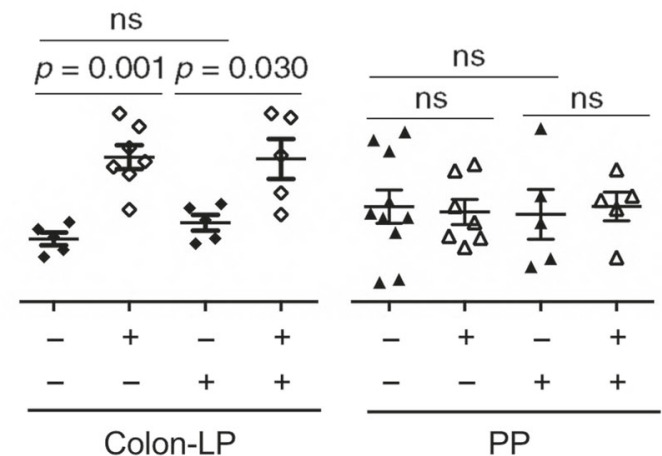

Fig. 9 Neonatal p40 treatment promotes induction of intestinal Treg in adult mice. Wt mice were treated with p40 from postnatal day 2 to day 21, as described in Fig. 2. Colitis was induced by TNBS in 7-week-old mice, as described in Fig. 7. Lymphocytes were isolated from lamina propria (LP) of the small intestine (SI) and the colon and Peyer's patches (PP). CD4, Foxp3, and CD25 expressing cells were assessed using flow cytometry analysis. Lymphocytes were gated for CD4 and then expression of Foxp3 and CD25 in CD4 ${ }^{+}$cells were analyzed. a Representative CD4 histogram and contour plot of Foxp3 and CD25 are shown. Numbers in Q2 of contour plots represent percentages of CD4 ${ }^{+}$Foxp3 ${ }^{+}$CD2 $5^{+}$ in total LP and PP cells. b The percentages of CD4 ${ }^{+} \mathrm{Foxp}^{+} \mathrm{CD} 25^{+}$cells in total LP and PP cells are shown. In no-p40 SI-LP and colon-LP groups without TNBS, cells from two mice per sample. In other groups, cells from one mouse per sample

without p40 treatment was $84.34 \pm 18.25$ and $85.23 \pm 17.15 \mu \mathrm{m}$ in this experiment, respectively. Only finger-like projections that extend into the lumen of the small intestine with the length longer than $22 \mu \mathrm{m}$ were counted.

Detection of $\mathrm{p} 40$ release from hydrogels in the intestinal tract in mice

p40 was labeled with NHS-rhodamine (Pierce Biotechnology, Rockford, IL), according to the manufacturer's instructions. Fourteen-day-old mice were gavaged with hydrogels containing NHS-rhodamine-p40 at $10 \mu \mathrm{g} /$ mouse and euthanized 2, 4, $6 \mathrm{~h}$ after gavage. Mice received hydrogels without $p 40$ for $6 \mathrm{~h}$ were used as control. Paraffin-embedded tissue sections were prepared for observing an NHS-rhodamine-p40 using fluorescent microscope. Small intestinal and colonic mucosal tissues were isolated, solubilized in tissue lysate buffer (Sigma-Aldrich), and homogenized. After centrifugation, the supernatants of mucosal lysates were collected for examining fluorescent intensity using a plate reader. The NHS-rhodamine-p40 concentration in mucosal lysates was determined by comparing to the NHS-rhodamine-p40 concentration curve. Protein concentrations in mucosal lysates were determined using a BCA protein assay kit. p40 delivered to the tissues was presented as: $\mu \mathrm{g}$ p40/mg tissue lysates.

To detect phosphorylation of EGFR, small intestinal and colonic epithelial cells were isolated as previously described. ${ }^{16,17}$ After incubation of intestinal tissues with dithiothreitol and EDTA for releasing villi and crypts, epithelial cells were sorted using a biotinlabeled E-cadherin antibody and streptavidin magnetic beads. Cellular proteins were used for immunoprecipition of tyrosine phosphorylated proteins using phospho-tyrosine mouse monoclonal antibody (P-Tyr-100)-conjugated to sepharose beads (Cell Signaling Technology), according to the manufacturer's 
instruction. Western blot analysis of precipitated proteins was performed using a rabbit polyclonal anti-EGFR antibody (EMD Millipore Corporation).

\section{Induction and analysis of intestinal injury and colitis}

Two mouse models of colitis were induced in 6- to 7-week-old mice. Colonic tissue was collected for isolation of RNA, myeloperoxidase assay, preparing paraffin-embedded tissue sections. H\&E stained sections were used for light microscopic examination to assess colon injury and inflammation. Samples from the entire colon were examined by a pathologist blinded to treatment conditions.

In the DSS-induced injury and acute colitis model, mice were administered 3\% DSS (molecular weight 36-50 kDa; MP Biomedicals) in their drinking water for 4 days. Mice were euthanized at the end of DSS treatment for evaluation of intestinal injury and acute colitis. Mice were fed with drinking water as controls for DSS treatment. A modified combined scoring system, including degree of inflammation (scale of 0-3) and crypt damage (0-4), percentage of area involved by inflammation (0-4) and crypt damage (0-4), and depth of inflammation (0-3), was applied for assessing DSSinduced intestinal injury and colitis by DSS.

In TNBS-induced acute colitis model, mice were treated with $100 \mu \mathrm{l}$ of $70 \mathrm{mM}$ TNBS in 50\% ethanol intrarectally. Control mice received $100 \mu \mathrm{l}$ of $50 \%$ ethanol intrarectally. Mice were euthanized 4 days after TNBS treatment. The scoring system used to assess TNBS-induced colitis was modified from a previous scoring system $^{51,52}$; lamina propria mononuclear cell and polymorphonuclear cell infiltration, enterocyte loss, crypt inflammation, and epithelial hyperplasia were scored from 0 to 3, yielding an additive score between 0 (no colitis) and 15 (maximal colitis).

\section{ELISA analysis}

For detecting fecal lgA levels, feces were solubilized in phosphatebuffered saline (PBS), and homogenized. Fecal supernatants were incubated in 96-well plates that were coated with an anti-mouse IgA antibody (Sigma-Aldrich). Nonspecific protein binding was blocked by incubating plates with $1 \%$ of bovine serum albumin in PBS. Plates were then incubated with an fluorescein isothiocyanate-conjugated anti-mouse IgA antibody (SigmaAldrich). Fluorescent intensity was measured using a fluorescent plate reader. Purified mouse IgA (Sigma-Aldrich) was used for generating a standard concentration curve. The results were presented as $\mu \mathrm{g} \lg \mathrm{A} / \mathrm{g}$ feces. Since the amount of feces from each 2- and 3-week-old mouse was small, we combined feces from two to three mice in the same treatment group as one sample. Samples from adult mice were used as feces from one mouse/ sample.

\section{Flow cytometry analysis}

Cultured $\mathrm{Cd}^{+} \mathrm{T}$ cells, lymphocytes isolated from lamina propria of the small intestine and the colon, and Peyper's patches were labeled with PE-Cy5.5-anti-CD4 (BioLegend, San Diego, CA) and PE-anti-CD25 antibodies (BioLegend) by incubation for $1 \mathrm{~h}$ at room temperature. Then, cells were fixed and permeabilized using Mouse Foxp3 Buffer Set (BD Biosences, San Jose, CA), followed by Foxp3 staining using Alexa Fluor 488 anti-Foxp3 antibody (BD Pharmingen, San Diego, CA), according to the manufacturer's instruction. Cells were analyzed using multi-color flow cytometry to determine the percentage of positive cells using a BD LSRII system (BD Biosciences).

Statistical analysis

Statistical significance was determined by one-way ANOVA analysis for multiple comparisons and $t$-test for comparing data from two samples using Prism 6.0 (GraphPad Software, Inc., San Diego, CA). A $p$-value $<0.05$ was defined as statistically significant. All data are presented as mean \pm S.E.M.
Detailed methods for YAMC and MSIE cell culture and preparation of conditioned medium, isolation, culture, and treatment of $\mathrm{CD}^{+} \mathrm{T}$ cells, ELISA analysis of TGF $\beta$, isolation of lymphocytes from mouse lamina propria and Peyer's patches, myeloperoxidase assay, real-time PCR assay, and immunohistochemistry are provided in the Supplementary Methods.

\section{ACKNOWLEDGEMENTS}

This work was supported by National Institutes of Health (NIH) grant R01DK081134 and the Crohn's \& Colitis Foundation Senior Research Award (to F.Y.), NIH grants R01DK56008 and R01DK54993 and the Crohn's \& Colitis Foundation Senior Research Award (to D.B.P.), NIH grants R01DK58587, R01CA77955, and P01CA116087 (to R.M P.), and National Natural Science Foundation of China Grant 81372982 (to F.H.), and core services performed through Vanderbilt University Medical Center's Digestive Disease Research Center supported by NIH grant P30DK058404. Whole slide imaging was performed in the Digital Histology Shared Resource at Vanderbilt University Medical Center (www.mc.vanderbilt.edu/dhsr). We thank Dr. M. Kay Washington from Vanderbilt University Medical Center for examining intestinal tissue sections from mouse models of colitis.

\section{AUTHOR CONTRIBUTIONS}

X.S., L.L., R.M.P., S.A.A., D.J.M., K.T.W., F.H., D.B.P., and F.Y. designed research studies and analyzed data; X.S., L.L., and F.Y. conducted experiments and acquired data. X.S. R.M.P., S.A.A., D.B.P., and F.Y. wrote the manuscript.

\section{ADDITIONAL INFORMATION}

The online version of this article (https://doi.org/10.1038/s41385-018-0034-3) contains supplementary material, which is available to authorized users.

Competing interests: The authors declare no competing interests.

Publisher's note: Springer Nature remains neutral with regard to jurisdictional claims in published maps and institutional affiliations.

\section{REFERENCES}

1. Hooper, L. V. Bacterial contributions to mammalian gut development. Trends Microbiol. 12, 129-34 (2004).

2. Gensollen, T., lyer, S. S., Kasper, D. L. \& Blumberg, R. S. How colonization by microbiota in early life shapes the immune system. Science 352, 539-44 (2016).

3. Blanton, L. V. et al. Gut bacteria that prevent growth impairments transmitted by microbiota from malnourished children. Science 351, 6275 (2016).

4. Haberman, Y. et al. Pediatric Crohn disease patients exhibit specific ileal transcriptome and microbiome signature. J. Clin. Invest. 124, 3617-33 (2014).

5. Michail, S. et al. Alterations in the gut microbiome of children with severe ulcerative colitis. Inflamm. Bowel Dis. 18, 1799-1808 (2012).

6. Lopez-Serrano, P. et al. Environmental risk factors in inflammatory bowel diseases. Investigating the hygiene hypothesis: a Spanish case-control study. Scand. J. Gastroenterol. 45, 1464-71 (2010).

7. Hill, C. et al. Expert consensus document. The International Scientific Association for Probiotics and Prebiotics consensus statement on the scope and appropriate use of the term probiotic. Nat. Rev. Gastroenterol. Hepatol. 11, 506-14 (2014).

8. Preidis, G. A. et al. Probiotics, enteric and diarrheal diseases, and global health. Gastroenterology 140, 8-14 (2011).

9. Guandalini, S. Probiotics for prevention and treatment of diarrhea. J. Clin. Gastroenterol. 45(Suppl.), S149-S53 (2011).

10. Schwarzer, M. et al. Lactobacillus plantarum strain maintains growth of infant mice during chronic undernutrition. Science 351, 854-7 (2016).

11. Yan, F. et al. Neonatal colonization of mice with LGG promotes intestinal development and decreases susceptibility to colitis in adulthood. Mucosal Immunol. 10, 117-27 (2017).

12. Thomas, C. M. \& Versalovic, J. Probiotics-host communication: modulation of signaling pathways in the intestine. Gut Microbes 1, 148-63 (2010).

13. Vanderpool, C., Yan, F. \& Polk, D. B. Mechanisms of probiotic action: implications for therapeutic applications in inflammatory bowel diseases. Inflamm. Bowel Dis. 14, 1585-96 (2008)

14. Yan, F. et al. Soluble proteins produced by probiotic bacteria regulate intestinal epithelial cell survival and growth. Gastroenterology 132, 562-75 (2007).

15. Wang, L. et al. Activation of epidermal growth factor receptor mediates mucin production stimulated byp40, a Lactobacillus rhamnosus GG-derived protein. J. Biol. Chem. 289, 20234-44 (2014). 
16. Wang, Y. et al. An LGG-derived protein promotes IgA production through upregulation of APRIL expression in intestinal epithelial cells. Mucosal Immunol. 10, 373-84 (2017).

17. Yan, F. et al. Colon-specific delivery of a probiotic-derived soluble protein ameliorates intestinal inflammation in mice through an EGFR-dependent mechanism. J. Clin. Invest. 121, 2242-53 (2011).

18. Yan, F. et al. A Lactobacillus rhamnosus GG-derived soluble protein, p40, stimulates ligand release from intestinal epithelial cells to transactivate epidermal growth factor receptor. J. Biol. Chem. 288, 30742-51 (2013).

19. Yarden, Y. The EGFR family and its ligands in human cancer: signalling mechanisms and therapeutic opportunities. Eur. J. Cancer 37(Suppl. 4), S3-S8 (2001).

20. Yarden, Y. \& Sliwkowski, M. X. Untangling the ErbB signalling network. Nat. Rev. Mol. Cell Biol. 2, 127-37 (2001).

21. Miettinen, P. J. et al. Epithelial immaturity and multiorgan failure in mice lacking epidermal growth factor receptor. Nature 376, 337-41 (1995).

22. Hirai, C. et al. Trophic effect of multiple growth factors in amniotic fluid or human milk on cultured human fetal small intestinal cells. J. Pediatr. Gastroenterol. Nutr. 34, 524-28 (2002).

23. Nojiri, T. et al. Clinical significance of amphiregulin and epidermal growth factor in colostrum. Arch. Gynecol. Obstet. 286, 643-47 (2012).

24. Michalsky, M. P., Lara-Marquez, M., Chun, L. \& Besner, G. E. Heparin-binding EGFlike growth factor is present in human amniotic fluid and breast milk. J. Pediatr. Surg. 37, 1-6 (2002)

25. Cheung, Q. C. et al. Generation of epidermal growth factor-expressing Lactococcus lactis and its enhancement on intestinal development and growth of early-weaned mice. Am. J. Clin. Nutr. 89, 871-79 (2009).

26. Sinha, A., Nightingale, J., West, K. P., Berlanga-Acosta, J. \& Playford, R. J. Epidermal growth factor enemas with oral mesalamine for mild-to-moderate left-sided ulcerative colitis or proctitis. N. Engl. J. Med. 349, 350-57 (2003).

27. Dube, P. E. et al. Epidermal growth factor receptor inhibits colitis-associated cancer in mice. J. Clin. Invest. 122, 2780-92 (2012).

28. Berni Canani, R. et al. Formula selection for management of children with cow's milk allergy influences the rate of acquisition of tolerance: a prospective multicenter study. J. Pediatr. 163, 771-7 e771 (2013).

29. Seth, A., Yan, F., Polk, D. B. \& Rao, R. K. Probiotics ameliorate the hydrogen peroxide-induced epithelial barrier disruption by a PKC- and MAP kinasedependent mechanism. Am. J. Physiol. Gastrointest. Liver Physiol. 294, G1060-G9 (2008).

30. Yoda, K. et al. Lactobacillus GG-fermented milk prevents DSS-induced colitis and regulates intestinal epithelial homeostasis through activation of epidermal growth factor receptor. Eur. J. Nutr. 53, 105-15 (2014).

31. Patel, R. M. et al. Probiotic bacteria induce maturation of intestinal claudin 3 expression and barrier function. Am. J. Pathol. 180, 626-35 (2012).

32. Cerutti, A. \& Rescigno, M. The biology of intestinal immunoglobulin $A$ responses. Immunity 28, 740-50 (2008).
33. Talham, G. L., Jiang, H. Q., Bos, N. A. \& Cebra, J. J. Segmented filamentous bacteria are potent stimuli of a physiologically normal state of the murine gut mucosal immune system. Infect. Immun. 67, 1992-2000 (1999).

34. Strober, W., Fuss, I. J. \& Blumberg, R. S. The immunology of mucosal models of inflammation. Annu. Rev. Immunol. 20, 495-549 (2002).

35. Okayasu, I. et al. A novel method in the induction of reliable experimental acute and chronic ulcerative colitis in mice. Gastroenterology 98, 694-702 (1990).

36. Pabst, O. New concepts in the generation and functions of IgA. Nat. Rev. Immunol. 12, 821-32 (2012)

37. Johansson, M. E., Sjovall, H. \& Hansson, G. C. The gastrointestinal mucus system in health and disease. Nat. Rev. Gastroenterol. Hepatol. 10, 352-61 (2013).

38. Tanoue, T., Atarashi, K. \& Honda, K. Development and maintenance of intestinal regulatory T cells. Nat. Rev. Immunol. 16, 295-309 (2016).

39. Vahabnezhad, E., Mochon, A. B., Wozniak, L. J. \& Ziring, D. A. Lactobacillus bacteremia associated with probiotic use in a pediatric patient with ulcerative colitis. J. Clin. Gastroenterol. 47, 437-9 (2013).

40. Land, M. H. et al. Lactobacillus sepsis associated with probiotic therapy. Pediatrics 115, 178-1 (2005).

41. Troyer, K. L. et al. Growth retardation, duodenal lesions, and aberrant ileum architecture in triple null mice lacking egf, amphiregulin, and tgf-alpha. Gastroenterology 121, 68-78 (2001).

42. Xian, C. J. Roles of epidermal growth factor family in the regulation of postnatal somatic growth. Endocr. Rev. 28, 284-96 (2007).

43. Olszak, T. et al. Microbial exposure during early life has persistent effects on natural killer T cell function. Science 336, 489-93 (2012).

44. Cox, L. M. et al. Altering the intestinal microbiota during a critical developmental window has lasting metabolic consequences. Cell 158, 705-21 (2014).

45. Rogier, E. W. et al. Secretory antibodies in breast milk promote long-term intestinal homeostasis by regulating the gut microbiota and host gene expression. Proc. Natl. Acad. Sci. USA 111, 3074-9 (2014).

46. Chen, W. et al. Conversion of peripheral CD4+CD25- naive T cells to CD4+CD25+ regulatory T cells by TGF-beta induction of transcription factor Foxp3. J. Exp. Med. 198, 1875-86 (2003).

47. Floch, M. H. et al. Recommendations for probiotic use-2015 update: proceedings and consensus opinion. J. Clin. Gastroenterol. 49(Suppl. 1), S69-S73 (2015).

48. Sanders, M. E. et al. An update on the use and investigation of probiotics in health and disease. Gut 62, 787-96 (2013).

49. Maldonado, J. et al. Human milk probiotic Lactobacillus fermentum CECT5716 reduces the incidence of gastrointestinal and upper respiratory tract infections in infants. J. Pediatr. Gastroenterol. Nutr. 54, 55-61 (2012).

50. Marzuillo, P., Guarino, S. \& Perrone, L. Atopic eczema could be a cause and not an effect of cow's milk protein allergy. J. Pediatr. Gastroenterol. Nutr. 58, e23 (2014).

51. Fuss, I. J. et al. Anti-interleukin 12 treatment regulates apoptosis of Th1 T cells in experimental colitis in mice. Gastroenterology 117, 78-1088 (1999).

52. Neurath, M. F. et al. The transcription factor T-bet regulates mucosal T cell activation in experimental colitis and Crohn's disease. J. Exp. Med. 195, 1129-43 (2002). 